\title{
A Novel Receiving and Extraction Method for Echoes of Multistatic Sonar
}

\author{
Lanyong Zhang, Yixuan Du, and Bing Li \\ College of Automation, Harbin Engineering University, Harbin, Heilongjiang 150001, China \\ Correspondence should be addressed to Lanyong Zhang; zhanglanyong@126.com
}

Received 4 August 2014; Accepted 13 October 2014; Published 21 December 2014

Academic Editor: Jingjing Zhou

Copyright (C) 2014 Lanyong Zhang et al. This is an open access article distributed under the Creative Commons Attribution License, which permits unrestricted use, distribution, and reproduction in any medium, provided the original work is properly cited.

Multiple carrier frequency detecting signals are transmitted simultaneously by multiple transmitters in multistatic sonar. The echoes mixed with different carrier frequency in the receiver. The different carrier frequency echoes must be separated from one another before features of echoes are extracted in the receiver. Such a problem can be solved by band-pass and low-pass filters. But the amount of operation by this way is too large for real-time realization. Thus this paper presents the technique of channelized receiver based on multiphase filter and the receiving schemes of echo. The proposed receiver has a smaller amount of operation compared to low-pass filter. At last, the feature extraction technology correlation processing and FDWT are introduced. In order to verify the feasibility of this scheme in multistatic sonar, the extracted features of original echo are contrasted with those of processed echo via simulation. Simulation results show that the proposed receiver provides considerable performance.

\section{Introduction}

Multistatic sonar is different from monostatic sonar, which places the source and receiver in the same location and is usually used as hull mounted sonar [1-3]. The transmitter transmits single-carrier detecting signal at one time in monostatic sonar, and so only single-carrier echo is considered at receiver when the returned signals are processed. A bistatic active sonar system is a generalization of the traditional monostatic active sonar to the case where the transmitter and receiver are not collocated. A multistatic sonar system is a further generalization to the case of multiple active sources and multiple receivers which are capable of receiving transmissions and target returns from any sources $[4,5]$. But in this paper the multistatic sonar consisted of multiple active transmitters and single receiver. To distinguish the characteristics of the transmitters, they must transmit different carrier signals simultaneously when the system works. Because of that, different carrier echoes exist at the receiver. This paper did a research on the receiving method for different carrier echoes.

\section{Problem Statement}

Linear frequency modulated signal is used in the transmitters of multistatic sonar system [6]. The pulse width is $T$, and the width of frequency modulation is $F$. Then the emission signal of source $k$ can be described as

$$
\begin{aligned}
& s_{k}(t)=a \cdot \cos \left(2 \pi f_{k} t+\pi \mu t^{2}\right), \\
& k=0 \sim N-1, \quad t \in\left[-\frac{T}{2}, \frac{T}{2}\right],
\end{aligned}
$$

where $\mu=F / T$ is chirp rate; $f_{k}$ is carrier frequency of source $k ; N$ is the amount of sources.

Figure 1 shows the geometric position of sources, receiver, and the target. $L$ represents the distance between the sources and receiver; $L_{2}$ represents the distance between source $A$ and target; $L_{1}$ represents the distance between source $\mathrm{B}$ and target; $L_{3}$ represents the distance between receiver and target; and $L_{0}$ represents the distance of sources. Then we can get the mathematical expression of echoes at the receiver as follows:

$$
s_{\text {echo }}(t)=\sum_{k=1}^{N} a \cdot \frac{e^{i k r_{k}}}{r_{k}} \cos \left[2 \pi f_{k}\left(t-\tau_{k}\right)+\pi \mu\left(t-\tau_{k}\right)^{2}\right],
$$

where $k=0 \sim N-1$, and $\tau_{k}$ represents the time-delay. $e^{i k r_{k}} / r$ is attenuation factor of channel transmission.

Echoes, which contain the features of target, are the returned emission signal reflected by the underwater targets. 


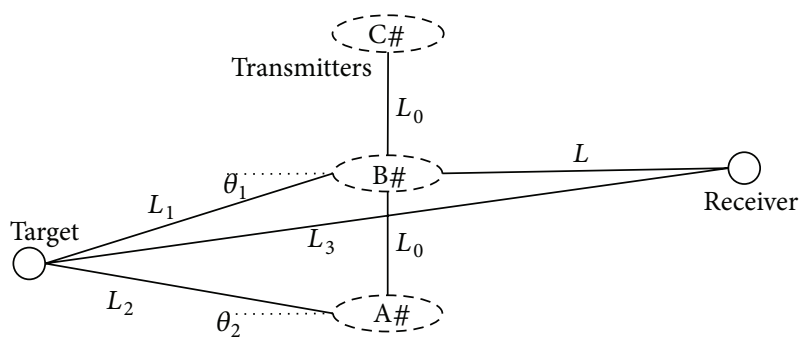

FIGURE 1: Schematic of multistatic sonar system.

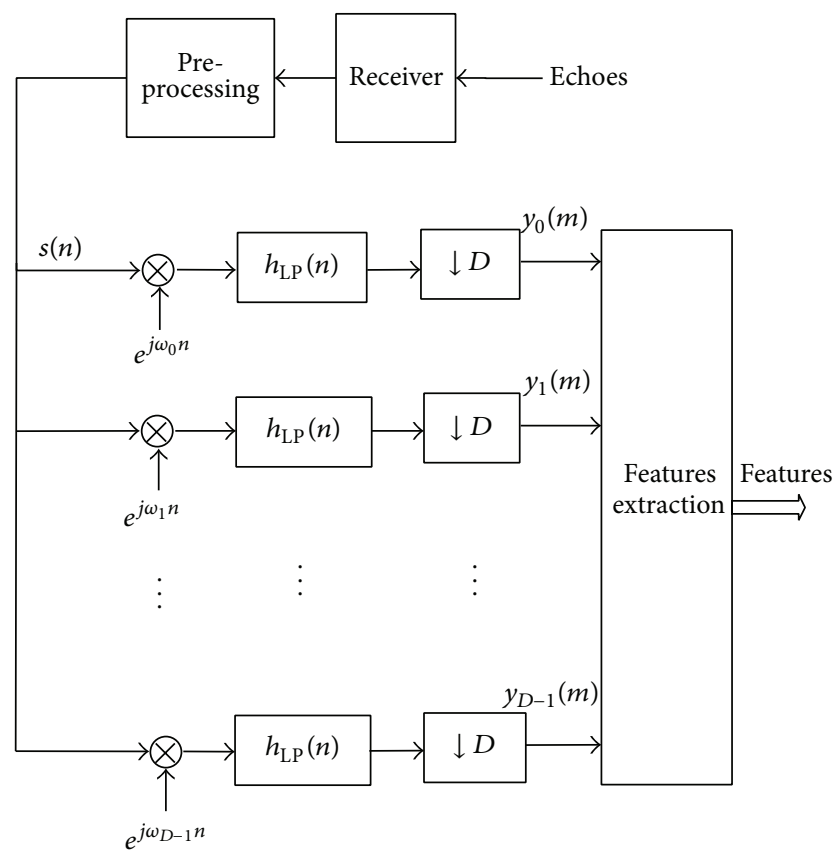

Figure 2: Scheme of signal separation based on low-pass filter banks.

To extract the features, the different carrier frequency echoes must be separated from one another. It can be solved by band-pass and low-pass filters. Figure 2 illustrates the scheme of signal separating process. Echoes are divided into many subfrequency bands, in which the low-pass digital filter banks are used in this method. Its structure is simple compared to the band-pass digital filter banks [7]. But this method requests that the low-pass filter has a so high rectangle coefficient and a so big order that the output would not contain other frequency components. It can be realized that the method had heavy computation and low efficiency when there are many channels.

\section{Channelized Receiver Based on Multiphase Filter Banks}

The whole sampling band $\left(0 \sim f_{s}\right)$ is divided into many parallel channels by the channelized receiver. The signals can be captured and demodulated whenever and wherever they appear (that is to say no matter which channel the signal appears in). The channelized receiver based on low-pass filter is difficult to imply in real-time implementation because the order of low-pass filter is too high to process the high data rate in time, while the multiphase filter technology is a more efficient method for channelized receiver [8].

$h(n)$ is the frequency response of the low-pass filter in Figure 2. So the output of channel $k$ can be given by

$$
\begin{aligned}
y_{k}(m) & =\left.\left\{\left[s(n) e^{j \omega_{k} n}\right] * h(n)\right\}\right|_{n=m D} \\
& =\sum_{i=-\infty}^{\infty} s(m D-i) e^{j \omega_{k}(m D-i)} \cdot h(i) \\
& =\sum_{p=0}^{D-1} \sum_{i=-\infty}^{\infty} s(m D-i D-p) e^{j \omega_{k}(m D-i D-p)} \cdot h(i D+p) .
\end{aligned}
$$

Define

$$
s_{p}(m)=s(m D-p), \quad h_{p}(m)=h(m D+p),
$$

where $s_{p}(m)$ is the multiphase component of original series $s(n)$ and $h_{p}(m)$ is the multiphase component of frequency response $h(n) . D$ is the number of channel. So (3) can be written as

$$
\begin{aligned}
y_{k}(m) & =\sum_{p=0}^{D-1} \sum_{i=-\infty}^{\infty} s_{p}(m-i) e^{j \omega_{k}(m D-i D-p)} \cdot h_{p}(i) \\
& =\sum_{p=0}^{D-1} x_{p}(m) e^{-j \omega_{k} p}
\end{aligned}
$$

where

$$
\begin{aligned}
x_{p}(m) & =\sum_{i=-\infty}^{\infty} s_{p}(m-i) e^{j \omega_{k}(m-i) D} \cdot h_{p}(i) \\
& =\left[s_{p}(m) e^{j \omega_{k} m D}\right] * h_{p}(m) .
\end{aligned}
$$

$\omega_{k}$ is the normalized frequency that ranks between $0 \sim$ $2 \pi$, and it can be expressed with $\omega_{k}=2 \pi k / D$. Then we can get

$$
\begin{gathered}
x_{p}(m)=\left[s_{p}(m) e^{j 2 \pi m k}\right] * h_{p}(m)=s_{p}(m) * h_{p}(m), \\
y_{k}(m)=\sum_{p=0}^{D-1}\left[x_{p}(m)\right] e^{-j(2 \pi / D) k p}=\operatorname{DFT}\left[x_{p}(m)\right] .
\end{gathered}
$$

Figure 3 shows the mathematical model of channelized receiver based on multiphase filter banks according to the equations derived above.

Figures 2 and 3 show that the multiphase filter has the same function with low-pass filter. The difference between them exists in engineering realization. The low-pass filter is composed of multiple mixers and a high-order filter. What is worse, it works under high data rate. The data rate is reduced by using multiphase filter banks because the $D$-extraction is located in front of the filter. The computation of the filter in Figure 3 is reduced to $1 / D$ of the computation in Figure 2. 


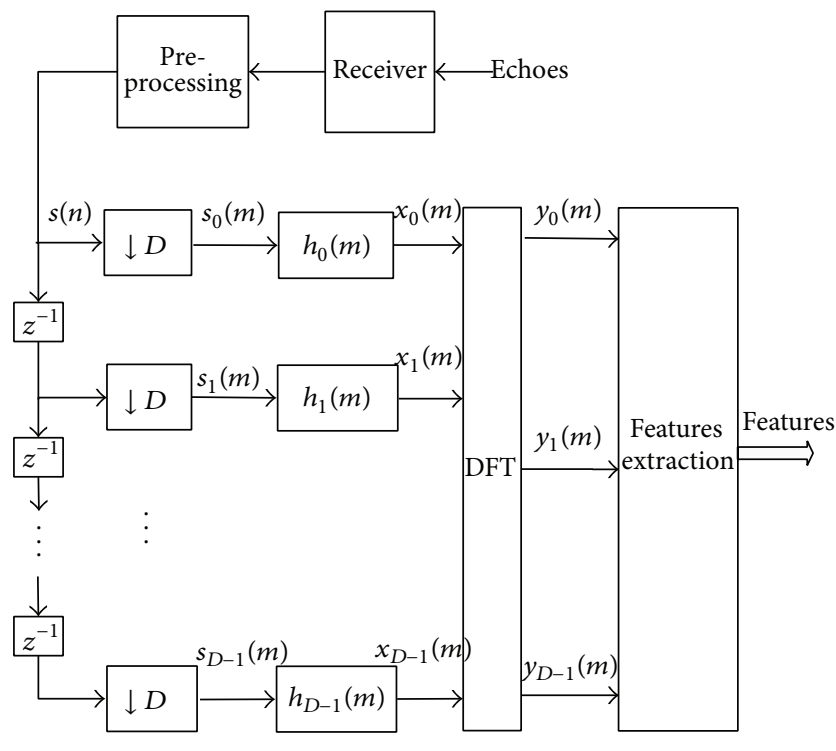

FIGURE 3: Scheme of signal separation based on multiphase filter banks.

The real-time processing capacity of this channelized receiver is sharply increased.

Given the length of $h_{\mathrm{LP}}(n)$ is $N$ in Figure 2, the multiplicative computation is $N$ when the receiver outputs one set of data from each channel. So the total computation is $D \times N$ in Figure 2. However with the structure of Figure 3, where the length of branch filter is $N / D$, the multiplicative computations of $D$ point DFT are about $\left(D \log _{2} D\right)$, and the total computation is $N+\left(D \log _{2} D\right)$. The amount of computation is reduced, which is beneficial to engineering realization, compared to Figure 2.

\section{Features Extraction}

The echoes are got by simulation according to the highlight model [9], which has the features of geometric highlight and elastic highlight. The features extraction in this paper is used to verify whether the features contained in the separated echoes are changed.

Correlation processing is a general signal processing method in the sonar technology [10]. The number, structure, and location of highlight are very important information in echoes. Obviously, the information can be got via correlation processing according to the definition of correlation and (1)(2). The cross-correlation function of emission signal and echo can be expressed as

$$
R_{k}(\tau)=\frac{e^{i k r_{k}}}{r_{k}} R^{\prime}\left(t-\tau_{k}\right), \quad k=0 \sim N-1,
$$

where $R^{\prime}(\tau)$ is the self-correlation function of emission signal.

Frequency discrete wavelet transform (FDWT) can eliminate geometric highlights, while keeping the elastic

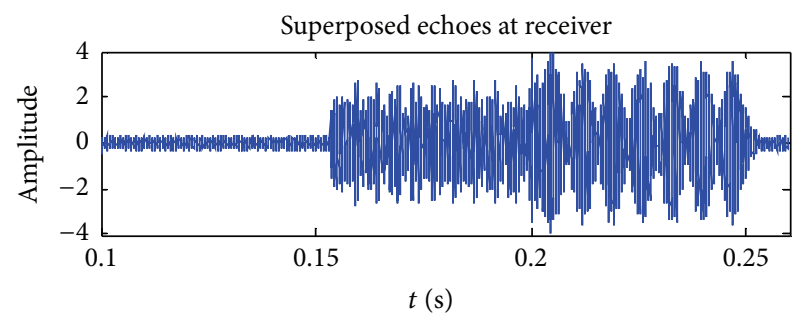

FIgURE 4: A section of echoes.

highlights. The analytic signal of echoes in each channel can be computed by

$$
y_{h k}(m)=y_{k}(m)+j \cdot \frac{2}{\pi} \sum_{k=-\infty}^{\infty} \frac{y(m-2 k-1)}{2 k+1} .
$$

The frequency domain signal can be given by the analytic signal. It is written as

$$
Y_{h k}(\omega)=\left|\sum_{m=0}^{Q-1} y_{h k}(m) \cdot e^{-j \omega m}\right| .
$$

$Q$ represents the dot number of FFT. And then the frequency domain signal is processed by discrete wavelet transform. The expression of discrete wavelet transform can be defined by

$$
\hat{W} T_{2^{k}}(\omega)=Y_{h k}(\omega) \cdot 2^{k / 2} e^{-j \omega t} \varphi\left(2^{k} \omega\right),
$$

where $\varphi(\omega)$ is the Fourier transformation of wavelet function $\varphi(t)$; and FDWT is realized according to the Mallat algorithm. The approximate wavelet-coefficients and detail waveletcoefficients of frequency domain signal are computed by

$$
\begin{gathered}
A_{0}\left[Y_{h k}(\omega)\right]=Y_{h k}(\omega), \\
A_{j}\left[Y_{h k}(\omega)\right]=\sum_{k} H(2 \omega-k) A_{j-1}\left[Y_{h k}(\omega)\right], \\
D_{j}\left[Y_{h k}(\omega)\right]=\sum_{k} G(2 \omega-k) A_{j-1}\left[Y_{h k}(\omega)\right] .
\end{gathered}
$$

$A_{j}$ is the approximate wavelet-coefficient in layer $j$ of signal $Y_{h k}(\omega) . D_{j}$ is the detail wavelet-coefficient in layer $j$ of signal $Y_{h k}(\omega)$. $H$ and $G$ are wavelet decompose filter. The elastic features are contained in the approximate waveletcoefficients of layer 4 , while geometric features are filtered in each detail wavelet-coefficient $[11,12]$.

\section{Simulation and Analysis}

Figure 4 illustrates a section of echoes got by simulation. The parameters of the simulation are set as follows. There are three transmitters, named A, B, and C. The distance between each of them is 130 meters. The emission signal is (1). The carriers are $40 \mathrm{kHz}, 60 \mathrm{kHz}, 80 \mathrm{kHz}, T=50 \mathrm{~ms}$, and $F=20 \mathrm{kHz}$. The sampling frequency is $200 \mathrm{kHz}$. The target is placed in 


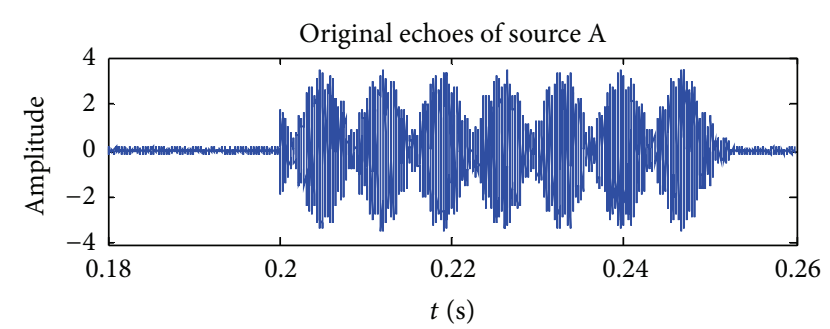

Figure 5: Original echoes of source A.

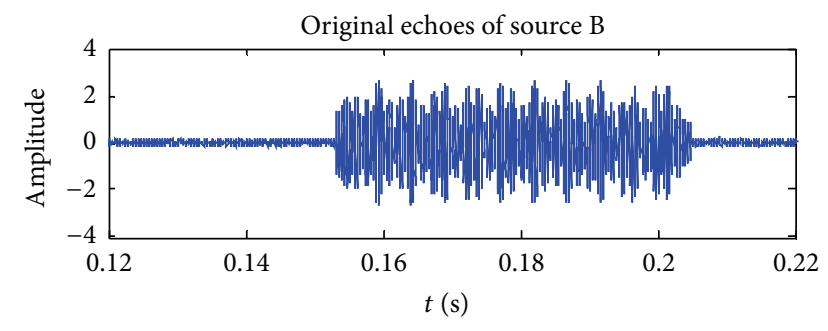

FIGURE 6: Original echoes of source B.

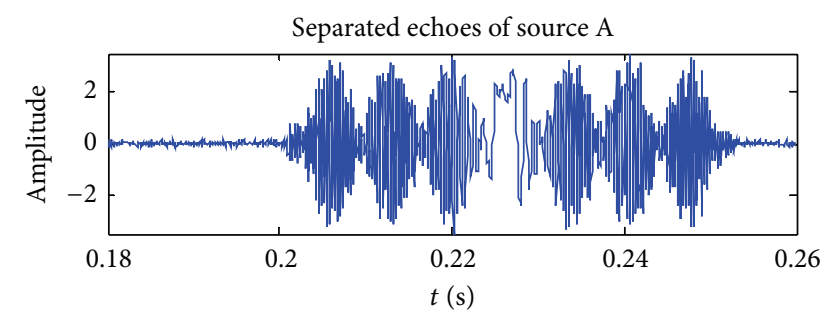

FIgURE 7: Separated echoes of source A.

the same plane with transmitters and receiver and has a 100-meter distance with source A while having a 65-meter distance with transmitter B. The target exists in the position that can be detected by both transmitter $\mathrm{A}$ and transmitter $B$. The emission signals of transmitters A and B are returned by target. Then they form one superposed signal with two kinds of echoes. Figure 5 illustrates the echo of transmitter A. Figure 6 illustrates the echo of transmitter B.

Figures 7 and 8 show the echoes of source A and source B separated by channelized receiver. The data rate of separated echoes is reduced because of the $D$ times extraction in channelized receiver.

Do the features contained in separated echoes change over the original echoes when the data rate is reduced? Figures 9-14 show the comparison of features between original echoes and separated echoes of source A and source $\mathrm{B}$, including the structure of highlight, geometric highlights and elastic highlights.

There are three highlights setting in original echoes when the simulation echoes are obtained. From Figures 9 and 10 we can get the conclusions that the number and the position of highlight keep invariant compared to original echoes.

The amplitude of the echoes' amplitude-frequency characteristics curve is associated with the amplitude of geometric highlight. The undulate of the echoes' amplitude-frequency

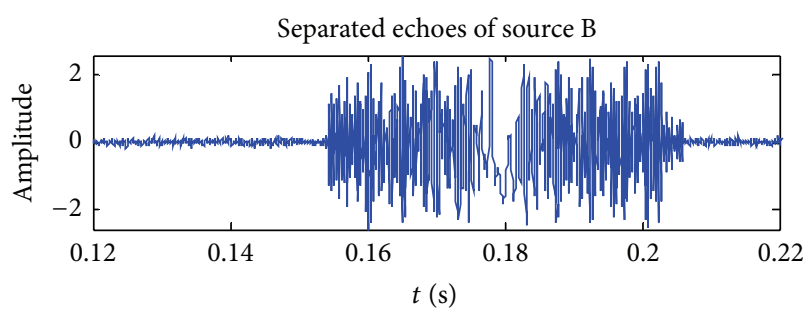

FIgURE 8: Separated echoes of source B.

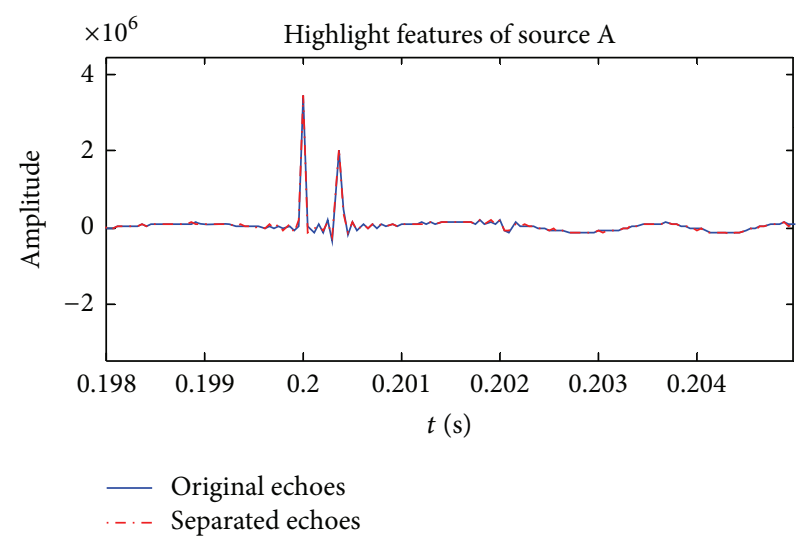

FIGURE 9: The comparison of highlight features extraction of source A.

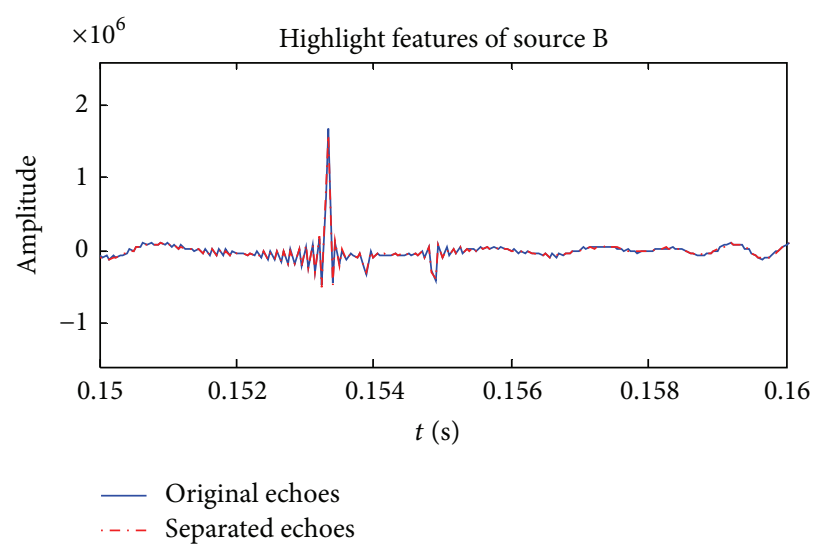

FIGURE 10: The comparison of highlight features extraction of source B.

characteristics curve envelop is associated with the timedelay of geometric highlight [12]. So the amplitude-frequency envelop-curves showed by Figures 11 and 12 represent the features of geometric highlight.

As mentioned above, the geometric highlight features can be filtered off and the elastic features are retained via FDWT. So the curves of the approximate wavelet-coefficients showed by Figures 13 and 14 represent the features of elastic highlight.

Table 1 shows the STD of errors of comparison curves of geometric highlight and elastic highlight. The value of STD of errors reflects the similarity degree of the comparison 


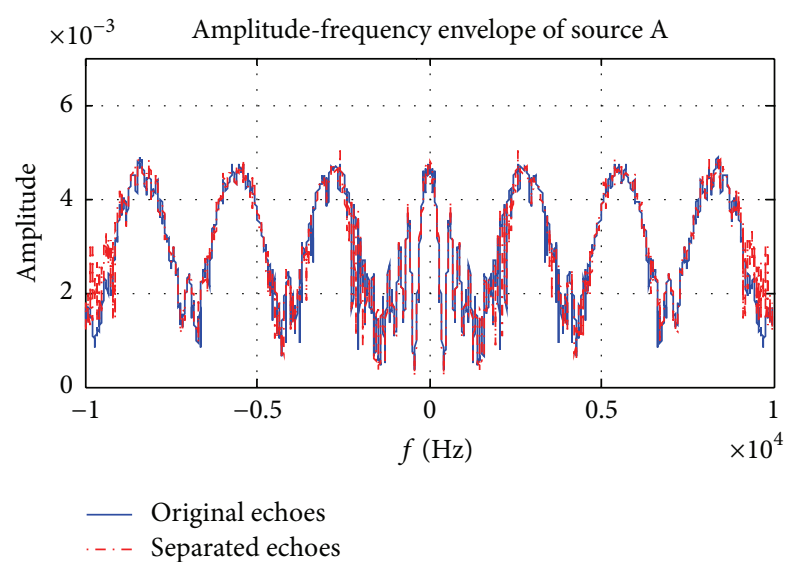

FIGURE 11: The comparison of amplitude-frequency envelope of source A.

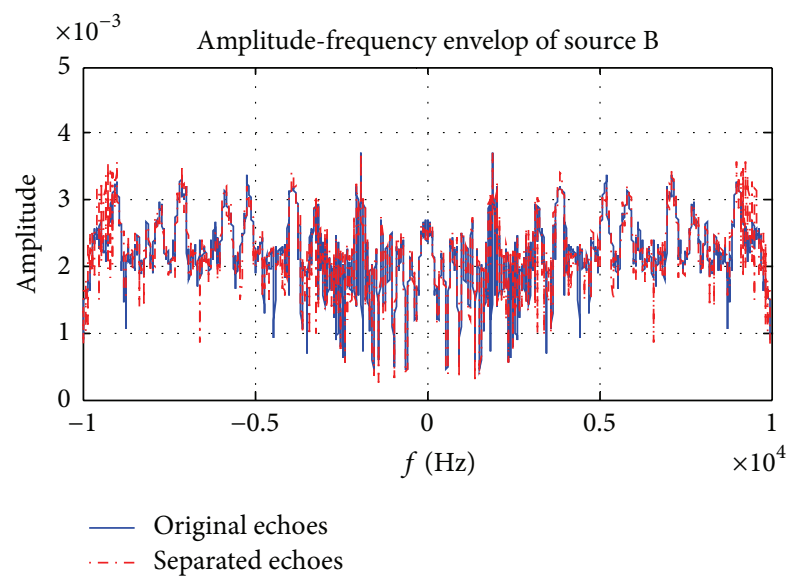

Figure 12: The comparison of amplitude-frequency envelope of source B.

TABLE 1: The STD of errors of comparison curves.

\begin{tabular}{lcc}
\hline STD & $\begin{array}{c}\text { Amplitude-frequency } \\
\text { envelop curves }\end{array}$ & $\begin{array}{c}\text { Approximate } \\
\text { wavelet-coefficients of FDWT }\end{array}$ \\
\hline Source A & $3.25 \times 10^{-4}$ & $7.45 \times 10^{-4}$ \\
Source B & $2.58 \times 10^{-4}$ & $3.72 \times 10^{-4}$ \\
\hline
\end{tabular}

curves. From Table 1 and Figures 9-14 we can conclude that the features contained in separated echoes keep invariant compared to original echoes.

\section{Conclusions}

Channelized receiving technology of software radio was applied to multistatic sonar system in this paper. Channelized receiver based on multiphase filter banks was used to separate the echoes produced by the emission signals of transmitter in multistatic sonar system. Through theoretical research and the comparison between the separated echoes with original echoes using the correlation processing results of simulation, we know that the features in separated echoes, such as

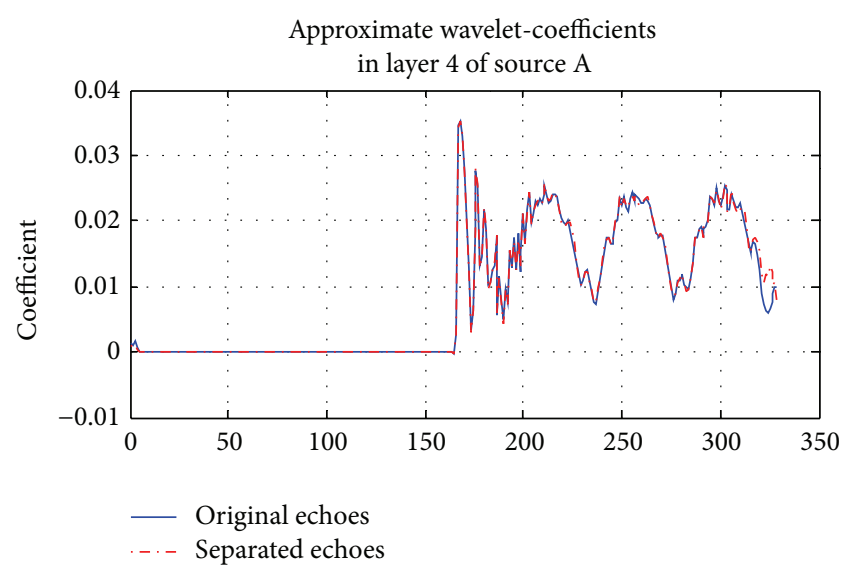

FIGURE 13: The comparison of elastic features extraction of source A.

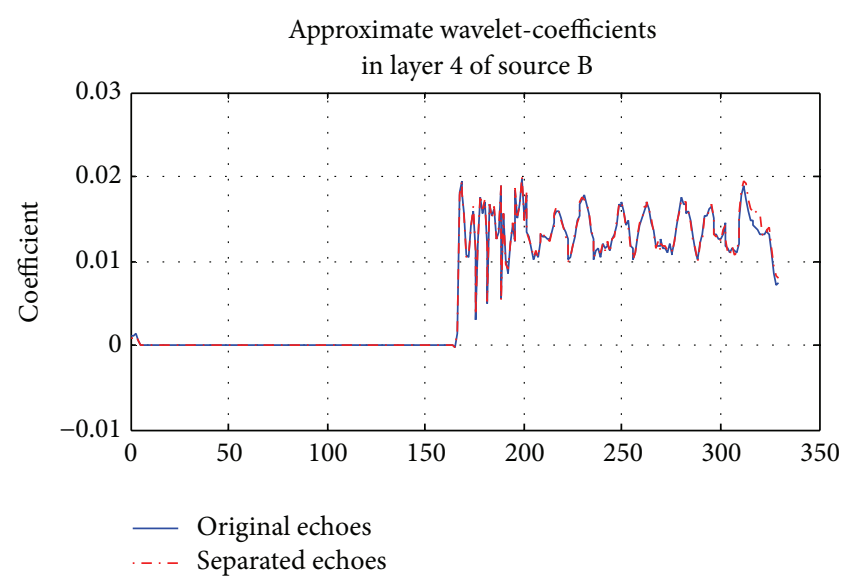

FIGURE 14: The comparison of elastic features extraction of source B.

the number, the position, the amplitude, the time-delay of highlight, amplitude-frequency characteristics, and FDWT, are not changed. The feasibility of using this method in multistatic sonar is verified. This method has an important guiding significance in engineering realization.

\section{Conflict of Interests}

The authors declare that there is no conflict of interests regarding the publication of this paper.

\section{Acknowledgments}

This work was supported in part by Research Fund for the Doctoral Program of Higher Education 20132304120015, China Postdoctoral Science Foundation Funded Project under no. 2012M510924, Heilongiiang Government Postdoctoral Foundation under no. LBH-12078, and Fundamental Research Funds for the Central Universities under HEUCFX41305. 


\section{References}

[1] S. Liu, Y. Du, and Z. Deng, "Study on robust output-tracking control of the sonar array," in Proceedings of the 6th World Congress on Intelligent Control and Automation (WCICA '06), pp. 2025-2028, Dalian, China, June 2006.

[2] B. Wang, "The nature of bistatic and multistatic radar," in Proceedings of the International Conference on Radar Proceedings, pp. 882-884, October 2001.

[3] K. Zhao, J. Liang, J. Karlsson, and J. Li, "Enhanced multistatic active sonar signal processing," The Journal of the Acoustical Society of America, vol. 134, no. 1, pp. 300-311, 2013.

[4] M. Daun and F. Ehlers, "Tracking algorithms for multistatic sonar systems," Eurasip Journal on Advances in Signal Processing, vol. 2010, Article ID 461538, 28 pages, 2010.

[5] S. Coraluppi, "Multistatic sonar localization," IEEE Journal of Oceanic Engineering, vol. 31, no. 4, pp. 964-974, 2006.

[6] D. W. Krout, W. L. J. Fox, and M. A. El-Sharkawi, "Probability of target presence for multistatic sonar ping sequencing," IEEE Journal of Oceanic Engineering, vol. 34, no. 4, pp. 603-609, 2009.

[7] R. Georgescu and P. Willett, "The GM-CPHD tracker applied to real and realistic multistatic sonar data sets," IEEE Journal of Oceanic Engineering, vol. 37, no. 2, pp. 220-235, 2012.

[8] D. Grimmett and C. Wakayama, "Multistatic tracking for continous active sonar using Doppler-bearing measurements," in Proceedings of the 16th International Conference of Information Fusion, pp. 258-265, Istanbul, Turkey, July 2013.

[9] E. Hanusa, D. W. Krout, and M. R. Gupta, "Contact clustering and fusion for preprocessing multistatic active sonar data," in Proceedings of the 16th International Conference of Information Fusion (FUSION '13), pp. 522-529, Istanbul, Turkey, July 2013.

[10] K. Zhao, J. Liang, J. Karlsson, and J. Li, "Enhanced multistatic active sonar signal processing," in Proceedings of the 38th IEEE International Conference on Acoustics, Speech, and Signal Processing (ICASSP '13), pp. 3861-3865, IEEE, Vancouver, Canada, May 2013.

[11] S. Steven, W. Peter, and B.-S. Yaakov, "Maximum likelihood probabilistic multi-hypothesis tracker applied to multistatic sonar data sets," in Proceedings of the Signal Processing, Sensor Fusion, and Target Recognition, pp. 8050-8055, Orlando, Fla, USA, April 2011.

[12] F. Ehlers, D. Orlando, and G. Ricci, "Batch tracking algorithm for multistatic sonars," IET Radar, Sonar and Navigation, vol. 6, no. 8, pp. 746-752, 2012. 


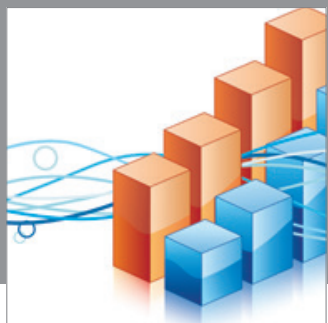

Advances in

Operations Research

mansans

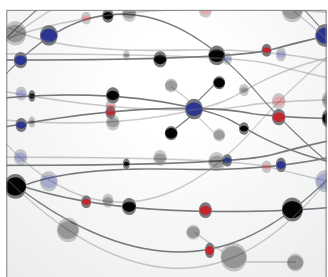

The Scientific World Journal
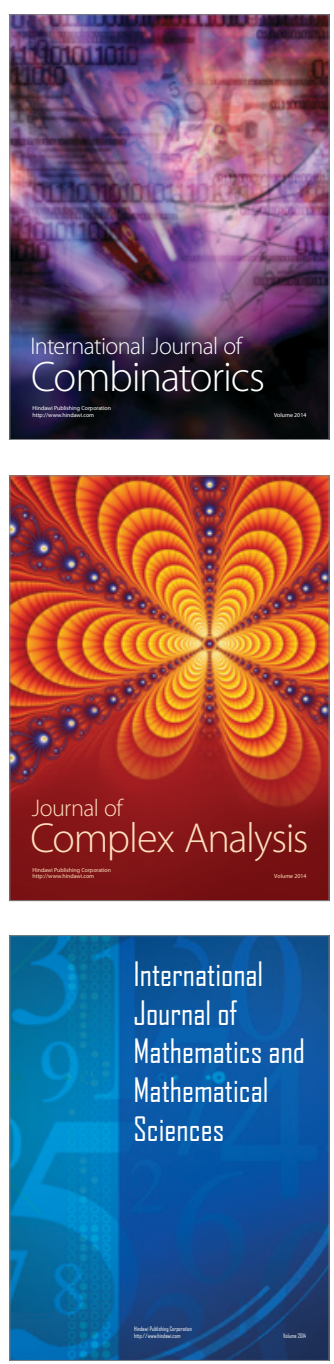
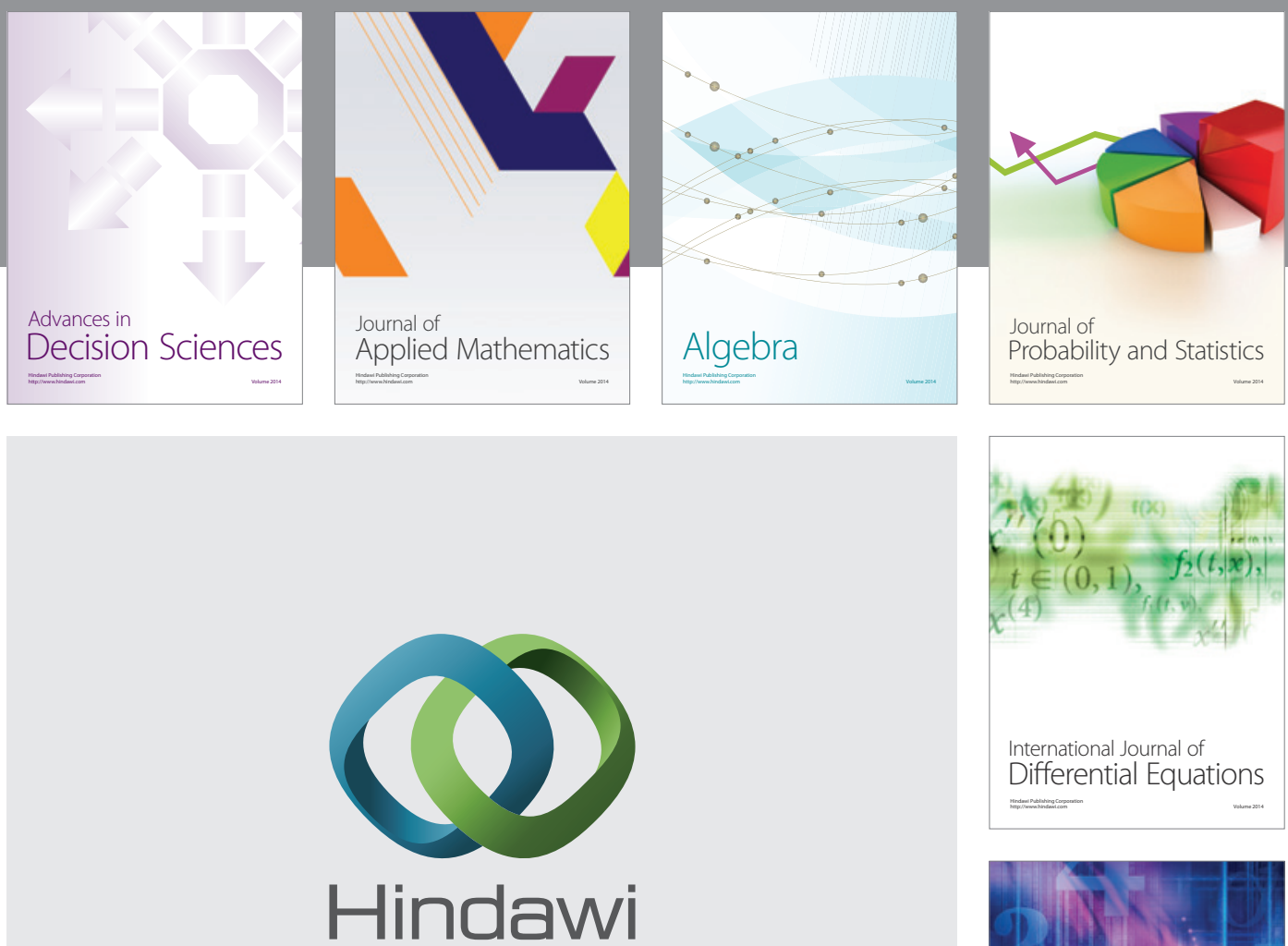

Submit your manuscripts at http://www.hindawi.com
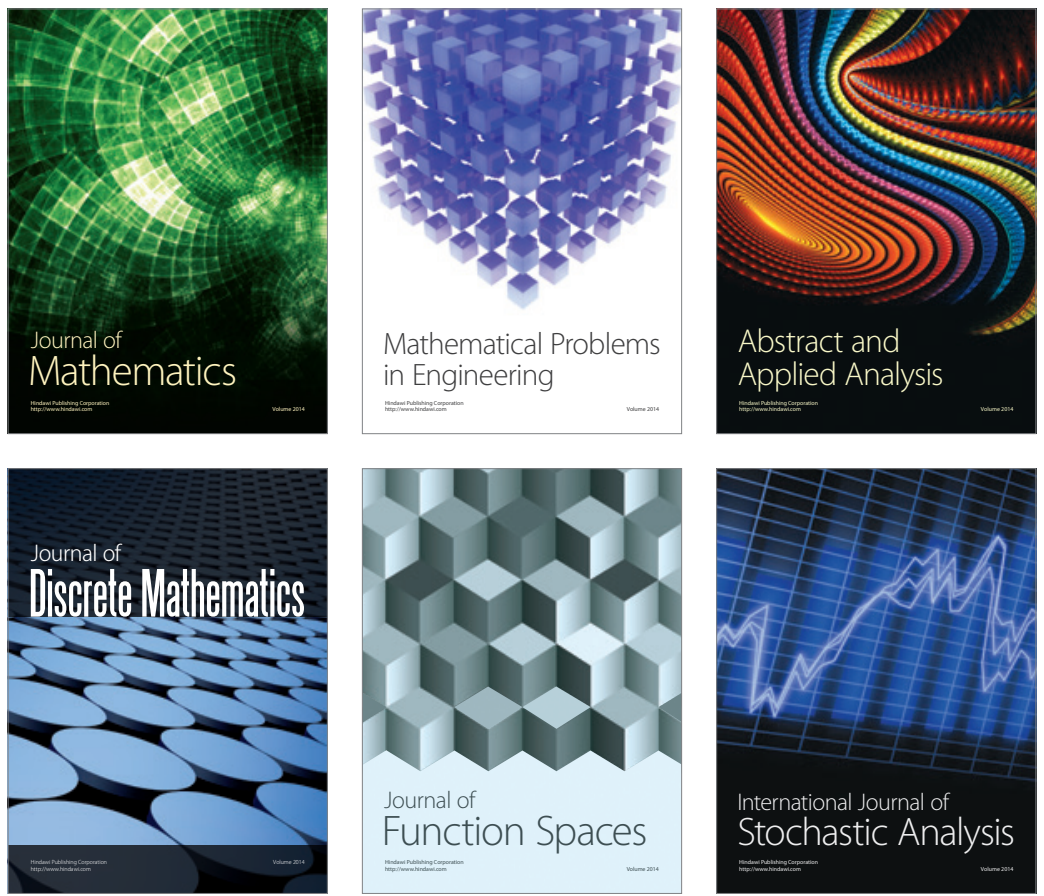

Journal of

Function Spaces

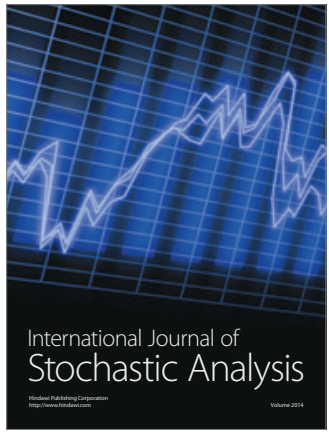

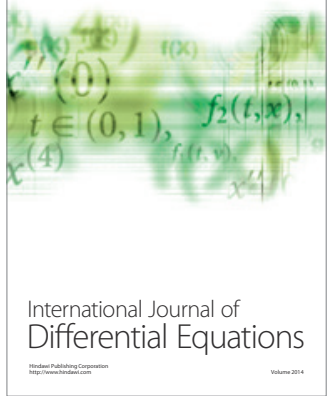
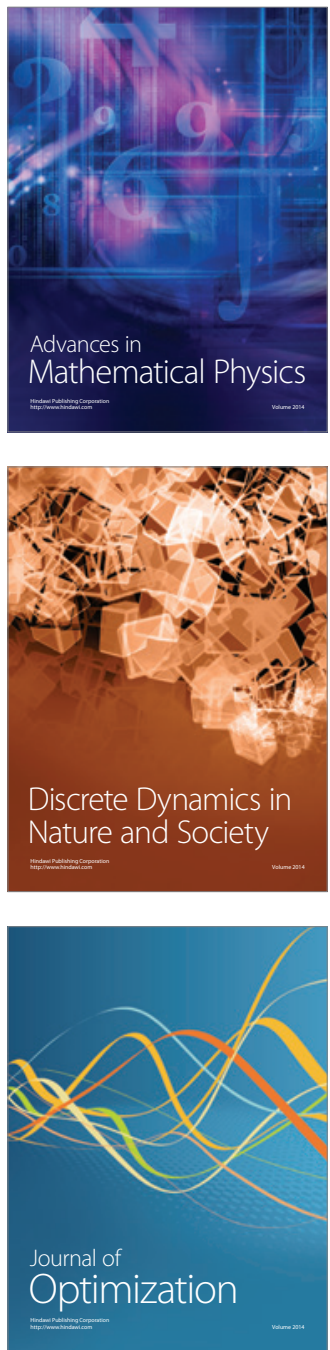